\title{
GRAVITATIONAL CAPTURE AND SCATTERING OF STRAIGHT TEST STRINGS WITH LARGE IMPACT PARAMETERS *
}

\author{
Don N. Page ${ }^{\dagger}$ \\ CIAR Cosmology Program, Institute for Theoretical Physics \\ Department of Physics, University of Alberta \\ Edmonton, Alberta, Canada T6G 2J1
}

(1998 April 30)

\begin{abstract}
The capture or scattering of an initially straight infinite test cosmic string by a Kerr-Newman black hole, or by any other small source of an electrovac gravitational field, is analyzed analytically when the string moves with initial velocity $v_{0}$ and large impact parameter $z_{0}=b \gg M$ so that the string stays very nearly straight (except during the final capture process, if that occurs, or except far behind the gravitating object, if $b \ngtr M / \sqrt{1-v_{0}^{2}}$ ). The critical impact parameter for capture at low velocities, $v_{0} \ll 1-Q^{2} / M^{2}$, is shown to be $b_{\text {crit }}=\sqrt{(\pi / 2)\left(M^{2}-Q^{2}\right) / v_{0}}+O(M)$. For all $b>b_{\text {crit }}$, the displacement of the string from the plane of the gravitating object after the scattering approaches the final value $z_{f} \approx \sqrt{b^{2}-(\pi / 2)\left(M^{2}-Q^{2}\right) / v_{0}}-2 \pi M v_{0} / \sqrt{1-v_{0}^{2}}$, for any $v_{0}$, so long as $b \gg M$.
\end{abstract}

*Alberta-Thy-05-98, gr-qc/9804088

†Internet address: don@phys.ualberta.ca 


\section{Introduction}

The scattering and capture of classical particles by black holes and other gravitating objects has been thoroughly analyzed, but the scattering and capturing of long cosmic strings has received much less attention [1, 2, 30, no doubt because the extension of the string makes it a much more difficult problem. In the ultrarelativistic limit of a string moving very near the speed of light in a direction perpendicular to the string, the propagation of disturbances along the string suffers a large time dilation, so that each piece of the string is effectively decoupled and moves very nearly along a null geodesic. Therefore, in this limit one can easily calculate the scattering of a string by a black hole (at least if one assumes that loops which may form in the string do not break off). For example, De Villiers and Frolov [2] find that the critical impact parameter for capture is the same as that of a photon, $3 \sqrt{3} M$ for the Schwarzschild metric.

However, for lower velocities, disturbances have time to propagate along the string, and so the problem becomes much more difficult. So far only numerical studies have been made of the capture of these slower strings by black holes [1, 2], and, until the final part of [3], which was done independently of this present paper and completed just shortly after my first rough draft, no one had found how the scattering behaves in the limit of very low velocity.

In this paper I shall show that the scattering of test strings can be calculated analytically when the strings stay sufficiently far from the gravitating object that they remain nearly straight. One finds that at sufficiently low velocities (depending upon the impact parameter), the scattering is dominated by terms quadratic in the mass and charge of the gravitating object. One can easily estimate the dependence of the critical impact parameter on the initial string velocity (going as its inverse square root). By a simple calculation that was a few orders of magnitude more difficult for me, one can also explicitly get the precise numerical coefficient.

\section{Power-law velocity dependence of the critical impact parameter}

First, let us get the power-law velocity dependence by a simple argument that does not require writing down any equations.

When the string is moving very slowly, then a long portion of it that is nearest the gravitating object will follow a sequence of nearly static configurations. Make the assumption that the string is everywhere far from the gravitating object compared with the gravitating object's intrinsic size (which for a black hole is of the order of the hole mass $M$, using units here and throughout this paper with $G=c=4 \pi \epsilon_{0}=1$, since the charge $Q$ and angular momentum $J=M a$ merely give fractional corrections to the size that are of order unity). Then at the location of the string, the Kerr- 
Newman (rotating, charged) black hole metric, or the metric of any gravitational source that is small compared with the distance to the string, can be adequately approximated by the static spherically symmetric Reissner-Nordstrom metric with the same mass $M$ and charge $Q$.

We shall consider here only a Nambu-Goto test string (i.e., a relativistic string with tension equal to its energy per unit length, $\mu$, in the frame of the string, both of which are negligibly small compared to all other relevant parameters in order that the string be a test string which does not affect the background spacetime geometry — this requires that $\mu \ll M \gamma_{0} v_{0} / b$ [4], where $M$ is the mass of the gravitating object, $v_{0}$ is the initial velocity of the string in the frame of the object, $\gamma_{0} \equiv 1 / \sqrt{1-v_{0}^{2}}$ is the relativistic gamma factor, and $b$ is the string impact parameter). Each static configuration of a Nambu-Goto test string in a static metric has a spatial configuration that follows a geodesic of an auxiliary spatial metric that is obtained from the spatial part of the actual spacetime metric by multiplying it by $-g_{00}$, the absolute square of the magnitude of the time translation Killing vector [5]. In the weak-field limit of a static metric with a nonrelativistic source (i.e., one with pressure much less than the energy density, including the rest mass energy density in the latter), the source of the linearized metric perturbation from the Minkowski flat spacetime metric is mainly the energy density $T_{00}$, and this gives rise to the trace-reversed metric perturbation $\bar{h}_{\mu \nu}$ that is dominated by the $\bar{h}_{00}$ term. When one reverses the trace of this to get the regular metric perturbation $h_{\mu \nu}$ and multiplies the resulting spatial part of the metric by $-g_{00}$, one gets an auxiliary spatial metric that is flat to first order in the dominant metric perturbations $h_{\mu \nu}$ that are generated by the dominant stress-energy component $T_{00}$. Hence a static string undergoes no bending to first order in a static weak-field metric of a nonrelativistic source [6].

By Birkhoff's theorem, the spherically symmetric vacuum (or electrovac, including the stress-energy tensor of a spherically symmetric electromagnetic field, when the charge $Q$ is nonzero) solution of Einstein's equations is the one-parameter $(M)$ static vacuum Schwarzschild metric when $Q=0$, or the two-parameter ( $M$ and $Q)$ static electrovac Reissner-Nordstrom metric [7]. Hence in the general spherically symmetric case in the weak-field limit (to which the general stationary Kerr-Newman metric is an adequate approximation at large distances where the field is weak), one gets the same result as for a weak-field static metric with a nonrelativistic source: static strings do not bend to first order in $M$, even for a black hole.

(Static neutral strings also do not bend to first order in $Q$, since the electromagnetic stress-energy tensor and Reissner-Nordstrom metric depend quadratically on $Q$. Like $M, Q$ has the dimension of length when $G=c=4 \pi \epsilon_{0}=1$ and has a magnitude no larger than $M$ for a black hole.)

One can also see this result directly for the Reissner-Nordstrom metric when one multiplies its spatial part by $-g_{00}$ and replaces the usual radial variable $r$ with a new radial variable that is $r-M$, since the resulting auxiliary spatial metric, where 
a static string follows a geodesic, is flat except for a correction term proportional to $M^{2}-Q^{2}$.

Therefore, the quasistatic sequence of string configurations that pass by at great distance and low velocity from a black hole or other small gravitational source are not bent to first order in $M$, but they are to second order (actually by an amount proportional to the correction term in the auxiliary spatial metric that is proportional to $M^{2}-Q^{2}$ ). If the string is not captured, its nearest distance from the gravitating object will be of the order of its impact parameter $b=z_{0}$ (the displacement of the plane of the string's initial motion from the gravitating object). When the nearest distance of the string from the gravitating object is of order $b$, by dimensional analysis it is obvious that the bending angle is proportional to $M^{2} / b^{2}$ (more precisely, to $\left(M^{2}-Q^{2}\right) / b^{2}$, but for simplicity I shall assume that $M^{2}-Q^{2}$ is of the same order as $M^{2}$, which it will be for a black hole unless it is very near an extremal Reissner-Nordstrom black hole that has $Q^{2}=M^{2}$, and which it will be in for a general gravitating object if the charge $Q$ of the object is not very large in comparison with its total mass-energy $M$ ).

The actual displacement of the string configurations from the straight ones they would follow in Minkowski spacetime in the absence of the gravitational field depends on how great a length of it is bent by an angle of order $M^{2} / b^{2}$. This will be the length along the string for which there has been time for causal influences to propagate (i.e., for the string to bend) during the time that the string passes at a distance of order $b$ from the gravitating object. Since the string is traveling at a speed of the order of its initial speed $v_{0}$, it takes a time of order $b / v_{0}$ to pass the region where it is of order its nearest distance from the gravitating object. With the bending perturbation traveling at nearly unit speed $(c=1)$ along the slowly moving string, a length of string of order $b / v_{0}$ is bent by an angle of order $M^{2} / b^{2}$, giving a displacement of the bent part of the string of order $M^{2} /\left(b v_{0}\right)$.

Assuming that the displacement is toward the gravitating object (which it is, when $M^{2}-Q^{2}>0$, as I shall generally assume), the string will not be captured if its impact parameter $b$ is greater than this bending displacement, which will be the case if $b^{2}$ is greater than some quantity of the order of $M^{2} / v_{0}$. However, the string will generally be captured (certainly in the black hole case) if the string bends down to reach the gravitating object, which we would expect to be the case when $b^{2}$ is smaller. Thus we can estimate the critical impact parameter for slow string capture to be of the order of $M / \sqrt{v_{0}}$ (or, more precisely, proportional to $\sqrt{\left(M^{2}-Q^{2}\right) / v_{0}}$ in the limit that the initial string speed $v_{0}$ is sufficiently small). This part of the argument can easily be done in one's head in a few moments of quiet reflection (or in semi-quiet reflection, as in washing dishes). Now comes the dirty work of finding the precise numerical coefficient, which took me about a month of algebra (between various other duties, including the previously-mentioned moments of semiquiet reflection), if one counts my various false starts. 


\section{Outline of the method and approximations}

The method for calculating the capture and scattering of infinitely long, nearly straight, test cosmic strings with large impact parameter $b$ will be to write down approximate expressions for the string motion in two overlapping domains around the gravitating object. In the inner domain, the string configuration at each moment of time is assumed to be very nearly straight and thus determined by the position of its nearest point to the gravitating object. In this domain one calculates the small bending of the string to first order in deviations of the metric and connection from flatness, evaluated at the zeroth order position of the nearly straight string. In the outer domain, at distances much larger than the impact parameter $b$ from the gravitating object, the metric is sufficiently flat that its effect on the string motion is negligibly different from that of Minkowski spacetime, and one simply has outgoing perturbations of the initially straight string configuration. The small bending of the string by the nonflat metric in the inner domain is taken to be the source of these outgoing perturbations in the approximately flat outer domain.

This approximation method only works when the string stays nearly straight and is not bent at large angles by the metric of the gravitating object, a condition which is satisfied when the string is always far from the object compared with the object size, which is of order $M$ for a black hole. (More strictly correct, for a string

with an arbitrary initial relativistic gamma-factor $\gamma_{0}=1 / \sqrt{1-v_{0}^{2}}$, when the impact parameter $b=z_{0}$ is large compared with the energy $M \gamma_{0}$ of the gravitating object in the initial string frame, since for $\gamma_{0} \geq b /(2 M) \gg 1$ one gets cusps and loops in the string [8]. Nevertheless, the cusps and loops develop only far behind the gravitating object, where it focuses the different pieces of the string to cross, and so even for large $M \gamma_{0} / b$ the string stays nearly straight while it is having a significant gravitational interaction with the object.)

However, the method does not require that the configuration of the string stay spatially near what its unperturbed motion would be if the gravitating object were absent: the point on the string that at each moment of time is nearest to the gravitating object can follow a trajectory that has large deviations from the straight line it would follow at constant velocity if the string were unperturbed. The only assumption is that at each moment the string configuration passing through this point (where the string is nearest the gravitating object) is nearly straight, with small bending perturbations (small in angle, not necessarily small in the total linear deviation from the unperturbed straight string configurations) propagating outward along the string at the speed of light in the nearly flat outer domain. In this way the range of applicability of the calculations presented here extends to lower initial velocities, $v_{0} \gg\left(M^{2}-Q^{2}\right) / b^{2}$ (in particular, to such low velocities that the string is captured), than the results obtained by a different method of calculation by De Villiers and Frolov [3]. 
Our results are in perfect agreement in the overlap region $\left(M^{2}-Q^{2}\right) / b^{2} \ll$ $\gamma_{0} v_{0} \ll b / M$, where $\gamma_{0} v_{0} \equiv v_{0} / \sqrt{1-v_{0}^{2}}$, the magnitude of the spatial component of the four-velocity, is what I propose be named the celerity (the magnitude of the spatial momentum per rest mass, i.e., the hyperbolic sine of the rapidity, as the ordinary three-velocity magnitude or speed is the hyperbolic tangent of the rapidity, and the energy per rest mass, $\gamma_{0}$ itself, is the hyperbolic cosine of the rapidity). In this overlap region where both methods work, the string is only slightly scattered and is nowhere near to being captured (which it would do at lower celerities, where the left hand inequality is not satisfied) and is also nowhere near to forming loops behind the string (which it would do at higher celerities [8], where the right hand inequality is not satisfied).

In both spatial domains in my method of calculation (the domain closer to the gravitating object, where the string is at each moment of time nearly where it would be if it were straight, and the domain farther away, where one can take spacetime to be flat), I am assuming that the string stays sufficiently far from the gravitating object (except for its final capture, which will not be analyzed here, only the conditions that lead to its being captured) that it is only slightly bent, and that it is adequate to consider the gravitating object metric only to quadratic order in its mass $M$ and charge $Q$, i.e., only to order $M^{2} / r^{2}$ and $Q^{2} / r^{2}$ for these dimensionless quantities. I shall only consider these quadratic terms when they persist at $v_{0}=0$, since otherwise they are dominated by terms that are first order in $M / r$ (which all are multiplied by factors that go to zero linearly in $v_{0}$ ). In other words, I shall consider only terms that are of the lowest nontrivial order in $M / r$ (or in $Q / r$, which is counted as being of the same order; the lowest nontrivial order of both of these is quadratic when $v_{0}$ is very small) and in $M v_{0} / r$.

One might also ask about the effect of the angular momentum $J=M a$ of the gravitating object. It has the dimension of squared length and is no greater than $M^{2}$ for a Kerr-Newman gravitating object, so I shall count it as potentially of order $M^{2}$ (like $Q^{2}$, since neither can be larger than $M^{2}$, though of course either or both could be much smaller, but I need not assume that either or both are much smaller). The angular momentum couples linearly to the velocity of the string at low velocities to give dimensionless terms of order $J v_{0} / r^{2}$ or $M^{2} v_{0} / r^{2}$, but since these terms are taken to be negligible compared with the $M v_{0} / r$ terms I am retaining, this coupling can be neglected in the approximations I am making.

There is also a velocity-independent effect (more strictly, an effect that persists when the string velocity is taken to zero) of the angular momentum upon the string motion (upon the quasistatic configurations of the strings [5]), but this starts quadratically in $J$ and hence gives dimensionless effects proportional to $J^{2} / r^{4}$, which is of order $M^{4} / r^{4}$ and hence will be neglected. Using the upper bound on the angular momentum of a Kerr-Newman black hole, one can see that $J^{2} / r^{4}$ is less than or equal to $M^{2}\left(M^{2}-Q^{2}\right) / r^{4}$ and hence is negligible compared with the $\left(M^{2}-Q^{2}\right) / r^{2}$ 
term I am keeping, when the string is at $r \gg M$ as I am assuming in order that the string bending be small. For other gravitating objects in which $J^{2} / r^{4}$ is not bounded thus, I am assuming that it is still negligible compared with $\left(M^{2}-Q^{2}\right) / r^{2}$.

The net result of this consideration of the angular momentum of the gravitating object is that I can neglect it for my considerations of strings propagating far from the gravitating object. (If the angular momentum is of order $M^{2}$, its effects would not be negligible on strings propagating near the gravitational radius of the gravitating object, but I am not considering such strings in this paper.)

If the gravitating object is not a stationary electrovac black hole and so can have multipole moments that are not fixed by its mass, charge, and angular momentum, I shall assume that the string stays far enough away from the object, in comparison with the object size, that one can neglect the effect of all of these multipole moments. For a cosmic string with $\mu \sim 10^{-6}$, which is not actually infinitesimal, it would be generally be problematic to stay sufficiently far from a nonrelativistic gravitating object and remain in the test string approximation, $\mu \ll M \gamma_{0} v_{0} / b$ [4], so in practice for such cosmic strings the test string approximation adopted in this paper would be adequate for capture or scattering only by black holes or other very compact relativistic objects, such as neutron stars. However, in this paper I shall continue to assume that the test string approximation is good, and that the string stays far enough from the gravitating object that its angular momentum and multipole moments have a negligible effect upon the motion of the string.

\section{Derivation of the equations of motion of the string location}

As a result of the assumptions and approximations above, it is an adequate approximation for my present purposes to use the unique spherically symmetric electrovac metric with the same mass $M$ and charge $Q$ as that of the actual gravitating object, namely, the Reissner-Nordstrom metric

$$
d s^{2}=-\left(1-\frac{2 M}{r}-\frac{Q^{2}}{r^{2}}\right) d t^{2}+\left(1-\frac{2 M}{r}-\frac{Q^{2}}{r^{2}}\right)^{-1} d r^{2}+r^{2} d \theta^{2}+r^{2} \sin ^{2} \theta d \varphi^{2} .
$$

Although this is a slight aside from the approach I shall actually use, it is instructive to consider the precise bending angle for all static string configurations in this metric. As mentioned above, these follow geodesics of the auxiliary spatial metric obtained by multiplying the spatial part of the static metric by $-g_{00}$. Or, for a stationary metric

$$
d s^{2}=g_{00}\left(x^{k}\right) d t^{2}+2 g_{0 i}\left(x^{k}\right) d t d x^{i}+g_{i j}\left(x^{k}\right) d x^{i} d x^{j},
$$

such as the Kerr-Newman metric, the auxiliary spatial metric, wherein static strings (strings which have their spatial coordinates $x^{k}$ stay fixed as the time coordinate 
$t=x^{0}$ evolves) follow geodesics, is [5]

$$
d s_{\text {aux }}^{2}=\left(g_{0 i} g_{0 j}-g_{00} g_{i j}\right) d x^{i} d x^{j} .
$$

However, the auxiliary spatial metric for the stationary Kerr-Newman metric differs (in the appropriate coordinate system) from the auxiliary spatial metric for the static Reissner-Nordstrom metric only by terms that are quadratic in the angular momentum $J$ and hence which are negligible at the distant locations at which I am generally considering the strings to move.

For the Reissner-Nordstrom metric (11) above, the auxiliary spatial metric (3) takes the form

$$
d s_{\mathrm{aux}}^{2}=d R^{2}+\left(R^{2}-M^{2}+Q^{2}\right)\left(d \theta^{2}+\sin ^{2} \theta d \varphi^{2}\right)
$$

where, to show more explicitly that this is flat up to the correction terms proportional to $M^{2}-Q^{2}$, I have shifted the radial coordinate to $R=r-M$. If a static string, which follows a geodesic of this auxiliary spatial metric (丑), has its minimum value of $R$ as $R_{0}$, then the bending angle is [5]

$$
2 \delta=\pi-2 k^{\prime} K(k)=\pi\left(\frac{1}{4} k^{2}+\frac{7}{64} k^{4}+\frac{17}{256} k^{6}+O\left(k^{8}\right)\right) \approx \frac{\pi\left(M^{2}-Q^{2}\right)}{4 R_{0}^{2}},
$$

where $K(k)$ is the complete elliptic integral of the first kind with modulus

$$
k=\frac{\sqrt{M^{2}-Q^{2}}}{R_{0}}
$$

and complementary modulus

$$
k^{\prime}=\sqrt{1-k^{2}}=\frac{\sqrt{R_{0}^{2}-M^{2}+Q^{2}}}{R_{0}} .
$$

Now let us return to the question of what the motion of a nearly straight string is far from a gravitating object, in a metric that at that great distance is adequately approximated by the Reissner-Nordstrom metric. In a general curved spacetime, the motion of a free infinitesimally thin relativistic cosmic test string with the NambuGoto action [9, 10] (proportional to the area of the worldsheet swept out by the string) obeys the equation of motion [11

$$
P_{\beta}^{\alpha} P_{\gamma ; \alpha}^{\beta}=0
$$

where $P_{\beta}^{\alpha}$ is the rank-two projection tensor into the tangent space of the string worldsheet. This is actually the form of the equation of motion of any free $n$ brane in curved spacetime [11], such as the 0-brane or free particle, for which Eq. (8) becomes the geodesic equation with then $P_{\beta}^{\alpha}=-u^{\alpha} u_{\beta}$ being the rank-one 
projection tensor to the tangent space of the particle worldline (the space of vectors parallel to the particle four-velocity $u^{\alpha}$ ).

Although Eq. (8) is written purely in terms of structures in the spacetime and does not require any parametrization of the string worldsheet, for my analysis below it is convenient to introduce null coordinates $(u, v)$ on the worldsheet itself, so that with coordinates $x^{\alpha}$ for the spacetime, the string worldsheet takes the form $x^{\alpha}=x^{\alpha}(u, v)$. In order that the coordinates $(u, v)$ be indeed null in the induced geometry on the string worldsheet, one must have the constraint equations

$$
g_{\alpha \beta} x_{, u}^{\alpha} x_{, u}^{\beta}=g_{\alpha \beta} x_{, v}^{\alpha} x_{, v}^{\beta}=0,
$$

where a comma denotes a partial derivative. Then Eq. (8) becomes

$$
x_{, u v}^{\alpha}+\Gamma_{\beta \gamma}^{\alpha} x_{, u}^{\beta} x_{, v}^{\gamma}=0,
$$

where $\Gamma_{\beta \gamma}^{\alpha}$ are the Christoffel symbols of the spacetime metric in the basis of spacetime coordinates $x^{\alpha}$.

It will be convenient to use spatial coordinates $x \equiv x^{1}, y \equiv x^{2}$, and $z \equiv x^{3}$, with $\rho \equiv\left(x^{2}+y^{2}+z^{2}\right)^{1 / 2}$ being a new radial variable (and with the superscripts in this last equation being exponents, unlike in the previous three equations in this sentence, where they are particular choices of the indices $i$ used to denote spatial indices $x^{i}$ ), and to choose these coordinates so that the spatial part of the Reissner-Nordstrom metric (11) is manifestly conformally flat. This requires that the previously used radial coordinates $r$ and $R$ be given by

$$
R=r-M=\rho+\frac{M^{2}-Q^{2}}{4 \rho} .
$$

In these new coordinates, the Reissner-Nordstrom metric takes the form

$$
\begin{aligned}
d s^{2} & =-e^{2 \phi+2 \psi} d t^{2}+e^{-2 \phi}\left(d x^{2}+d y^{2}+d z^{2}\right) \\
& =\left(1+\frac{M}{\rho}+\frac{M^{2}-Q^{2}}{4 \rho^{2}}\right)^{-2}\left(1-\frac{M^{2}-Q^{2}}{4 \rho^{2}}\right)^{2} d t^{2} \\
& +\left(1+\frac{M}{\rho}+\frac{M^{2}-Q^{2}}{4 \rho^{2}}\right)^{2}\left(d x^{2}+d y^{2}+d z^{2}\right)
\end{aligned}
$$

with

$$
\begin{gathered}
\phi=-\ln \left(1+\frac{M}{\rho}+\frac{M^{2}-Q^{2}}{4 \rho^{2}}\right) \approx-\frac{M}{\rho}, \\
\psi=\ln \left(1-\frac{M^{2}-Q^{2}}{4 \rho^{2}}\right) \approx-\frac{M^{2}-Q^{2}}{4 \rho^{2}} .
\end{gathered}
$$

When $M=Q=0$, this metric is obviously the flat Minkowski metric, and in it I shall take the unperturbed solution to be a straight string extended in the $x$ direction, moving with speed $v_{0}$ in the $y$-direction, and at impact parameter or height 
$z=b$ above the $x-y$ plane. With a suitable choice of null coordinates $(u, v)$ on the string worldsheet (each of which can be replaced by an arbitrary smooth monotonic function of itself to give a new null coordinate), the location of this unperturbed string worldsheet in the flat Minkowski metric can be given by

$$
\begin{aligned}
t & =t_{0} \equiv \frac{1}{2}(u+v) \\
x & =x_{0} \equiv \frac{1}{2} \gamma_{0}^{-1}(v-u) \\
y & =y_{0} \equiv \frac{1}{2} v_{0}(u+v) \\
z & =z_{0} \equiv b
\end{aligned}
$$

Here $\gamma_{0}=1 / \sqrt{1-v_{0}^{2}}$ is the usual relativistic gamma-factor. Note that the (constant) speed $v_{0}$ is not to be confused with the null coordinate $v$.

Now when $M$ and possibly also $Q$ are nonzero, I shall take the string worldsheet to start off with the form of Eqs. (15) - (18) at the infinite past, at $t_{0}=-\infty$ for all $x_{0}$. Then in the outer domain, $\rho \gg b$, where the spacetime curvature may be ignored in comparison with the curvature in the region $\rho \sim b$ that the part of the string with $\left|x_{0}\right| \lesssim b$ reaches at $\left|t_{0}\right| \lesssim b / v_{0}$, there will essentially just be outgoing flat-spacetime perturbations of the string for $u \gtrsim-b / v_{0}, v \gtrsim-b / v_{0}$.

In particular, the string worldsheet will have in the outer domain the approximate form

$$
\begin{aligned}
t & \approx t_{0}+\theta(v-u) T(u)+\theta(u-v) T(v), \\
x & \approx x_{0}+\theta(v-u) X(u)-\theta(u-v) X(v), \\
y & \approx y_{0}+\theta(v-u) Y(u)+\theta(u-v) Y(v), \\
z & \approx z_{0}+\theta(v-u) Z(u)+\theta(u-v) Z(v),
\end{aligned}
$$

where I have used the fact that the perturbations from the metric in the region $\rho \sim b$, and the string worldsheet, are symmetric under $x \rightarrow-x$, or under $u \leftrightarrow v$ on the string worldsheet. Here $\theta$ is the standard Heaviside step function, 0 for negative argument, $1 / 2$ for argument zero, and 1 for positive argument. Therefore, the right half of the string with $x>0(v>u)$ will have right-moving perturbations that are functions of $u$ only and which move at the speed of light rightward away from the region $\rho \sim b$ where they are generated, and the left half of the string with $x<0$ $(u>v)$ will have left-moving perturbations that are functions of $v$ only and which move at the speed of light leftward away from the region $\rho \sim b$ where they are generated.

In the inner domain $\rho \sim b$ where the metric deviations from flat spacetime generate the perturbations (bending) of the string, Eqs. (19) - (22) are not valid in its separation of the perturbations into left- and right-moving modes only. However, so long as $\rho \gg M$, as we have been assuming, the string will be sufficiently straight 
in this inner domain that to an excellent approximation we may calculate the metric deviations that generate the string perturbations as if they acted at the location of a straight string passing though the trajectory that follows the $x=0$ midpoint of the string, where $u=v=t_{0}$. Matching this straight string in the inner domain with Eqs. (19) - (22) in the outer domain gives in the inner domain

$$
\begin{aligned}
t & \approx t\left(t_{0}\right)=t_{0}+T\left(t_{0}\right) \\
x & \approx\left(1-\gamma_{0} \dot{X}\left(t_{0}\right)\right) x_{0} \equiv \frac{1}{2} \gamma^{-1}(v-u) \\
y & \approx y\left(t_{0}\right)=y_{0}+Y\left(t_{0}\right)=v_{0} t_{0}+Y\left(t_{0}\right) \\
z & \approx z\left(t_{0}\right)=z_{0}+Z\left(t_{0}\right)=b+Z\left(t_{0}\right)
\end{aligned}
$$

Here and henceforth, an overdot will denote a derivative with respect to $t_{0} \equiv(u+$ $v) / 2$ at $x=0$, where $t_{0}=u=v$. In particular, $\dot{t}=1+\dot{T}, \dot{y}=v_{0}+\dot{Y}$, and $\dot{z}=\dot{Z}$.

From the constraint equations (9) that $u$ and $v$ be null coordinates on the string worldsheet, one can see that, taking the metric to be approximately Minkowskian,

$$
\gamma=\gamma\left(t_{0}\right) \approx\left(\dot{t}^{2}-\dot{y}^{2}-\dot{z}^{2}\right)^{-1 / 2}
$$

As a results of these approximations that the string is nearly straight in the inner domain and takes the flat spacetime form with outgoing perturbations in the outer domain, determining the approximate configuration of the string worldline reduces to finding the three functions of one variable $T, Y$, and $Z$, or, equivalently, $t\left(t_{0}\right)$, $y\left(t_{0}\right)$, and $z\left(t_{0}\right)$. In order to get ordinary differential equations for them, use the string equations of motion in the coordinate form of Eq. (10), integrated over $x$ at fixed $t$.

Now using the approximate form of the string worldsheet as seen in the largescale view from the outer domain, given by Eqs. (19) - (22), one can integrate the various $x_{, u v}^{\alpha}$ in Eq. (10) over $x$ at fixed $t$ to get

$$
\begin{aligned}
\int d x t_{, u v} & \approx \frac{1}{2 \gamma} \dot{T}=\frac{1}{2 \gamma}(\dot{t}-1) \\
\int d x x_{, u v} & \approx 0 \\
\int d x y_{, u v} & \approx \frac{1}{2 \gamma} \dot{Y}=\frac{1}{2 \gamma}\left(\dot{y}-v_{0}\right), \\
\int d x z_{, u v} & \approx \frac{1}{2 \gamma} \dot{Z}=\frac{1}{2 \gamma} \dot{z} .
\end{aligned}
$$

To integrate the various $\Gamma_{\beta \gamma}^{\alpha} x_{, u}^{\beta} x_{, v}^{\gamma}$ in Eq. (10) over $x$ at fixed $t$, use the approximately straight form of the string in the inner region for evaluating the $u$ - and $v$-derivatives of $t(u, v), y(u, v)$, and $z(u, v)$ as functions purely of $t_{0}=(u+v) / 2$ in terms of how $t, y$, and $z$ behave at $x=0$ as functions of $t_{0}$. However, I shall 
use the constraint equations (9) to determine the $u$ - and $v$-derivatives of $x(u, v)$. In particular, I take

$$
\begin{aligned}
t_{, u} & =t_{, v} \approx \frac{1}{2} \dot{t} \\
x_{, u} & =-x_{, v} \approx-\frac{1}{2} \sqrt{e^{4 \phi+2 \psi} \dot{t}^{2}-\dot{y}^{2}-\dot{z}^{2}}, \\
y_{, u} & =y_{, v} \approx \frac{1}{2} \dot{y} \\
z_{, u} & =z_{, v} \approx \frac{1}{2} \dot{z} .
\end{aligned}
$$

Then one gets, using a prime on the exponents $\phi$ and $\psi$ in the first form of the metric (12) to denote a derivative with respect to the radial coordinate $\rho$,

$$
\begin{aligned}
& \Gamma_{\beta \gamma}^{0} x_{, u}^{\beta} x_{, v}^{\gamma} \approx \frac{\phi^{\prime}+\psi^{\prime}}{2 \rho} \dot{t}(y \dot{y}+z \dot{z}), \\
& \Gamma_{\beta \gamma}^{1} x_{, u}^{\beta} x_{, v}^{\gamma} \approx e^{4 \phi+2 \psi} \frac{2 \phi^{\prime}+\psi^{\prime}}{4 \rho} \dot{t}^{2} x, \\
& \Gamma_{\beta \gamma}^{2} x_{, u}^{\beta} x_{, v}^{\gamma} \approx e^{4 \phi+2 \psi} \frac{\psi^{\prime}}{4 \rho} \dot{t}^{2} y-\frac{\phi^{\prime}}{2 \rho} \dot{z}(z \dot{y}-y \dot{z}), \\
& \Gamma_{\beta \gamma}^{3} x_{, u}^{\beta} x_{, v}^{\gamma} \approx e^{4 \phi+2 \psi} \frac{\psi^{\prime}}{4 \rho} \dot{t}^{2} z+\frac{\phi^{\prime}}{2 \rho} \dot{y}(z \dot{y}-y \dot{z}) .
\end{aligned}
$$

These expressions apply at any radius for any static spherically symmetric metric, which can be put into the form of the first form of the metric (12). The only approximation is the approximation of Eqs. (32) - (35) for the $u$ - and $v$-derivatives of the spacetime coordinates $(t, x, y, z)$ on the string worldsheet as functions of the null coordinates $(u, v)$ on the string worldsheet.

Now we shall explicitly go to the Reissner-Nordstrom form of the metric (12) and assume that we are at $\rho \gg M>|Q|$. Then using the approximate expressions in Eqs. (13) and (14) for $\phi$ and $\psi$, we can set $e^{4 \phi+2 \psi} \approx 1$ and

$$
\begin{aligned}
\frac{\phi^{\prime}}{\rho} & \approx \frac{M}{\rho^{3}}, \\
\frac{\psi^{\prime}}{\rho} & \approx \frac{M^{2}-Q^{2}}{2 \rho^{4}} .
\end{aligned}
$$

Since $\rho \gg M$, we have $\phi^{\prime} \gg \psi^{\prime}$, so we shall ignore $\psi^{\prime}$ in any term where it is added to a nonzero multiple of $\phi^{\prime}$ with the same factors of $\dot{t}, \dot{y}$, and $\dot{z}$.

Just as we integrated the various $x_{, u v}^{\alpha}$ in Eq. (10) over $x$ at fixed $t$ to get Eqs. (28) - (31), now we can use Eqs. (40) and (42) to integrate the various $\Gamma_{\beta \gamma}^{\alpha} x_{, u}^{\beta} x_{, v}^{\gamma}$ in Eq. (10) over $x$ at fixed $t$. If we now define

$$
r \equiv \sqrt{y^{2}+z^{2}}=\sqrt{\rho^{2}-x^{2}},
$$


the approximate distance from the gravitating object to the nearest point (at $x=0$ ) on the string (not quite the same as the previous use of $r$ to denote a Schwarzschildean or circumferential radial coordinate, though to the approximations being made, the two definitions are virtually indistinguishable at the point on the string nearest the gravitating object), we obtain

$$
\begin{aligned}
\int d x \Gamma_{\beta \gamma}^{0} x_{, u}^{\beta} x_{, v}^{\gamma} & \approx \frac{M}{r} \dot{t} \dot{r} \\
\int d x \Gamma_{\beta \gamma}^{1} x_{, u}^{\beta} x_{, v}^{\gamma} & \approx 0, \\
\int d x \Gamma_{\beta \gamma}^{2} x_{, u}^{\beta} x_{, v}^{\gamma} & \approx \frac{\pi\left(M^{2}-Q^{2}\right)}{16 r^{3}} \dot{t}^{2} y-\frac{M}{r^{2}} \dot{z}(z \dot{y}-y \dot{z}), \\
\int d x \Gamma_{\beta \gamma}^{3} x_{, u}^{\beta} x_{, v}^{\gamma} & \approx \frac{\pi\left(M^{2}-Q^{2}\right)}{16 r^{3}} \dot{t}^{2} z+\frac{M}{r^{2}} \dot{y}(z \dot{y}-y \dot{z}) .
\end{aligned}
$$

Now if we combine Eqs. (43) - (46) with Eqs. (28) - (31) to get the $x$-integrated form of the string equations of motion (10), we get the following ordinary differential equations for the coordinates $t\left(t_{0}\right), y\left(t_{0}\right)$, and $z\left(t_{0}\right)$ of the point on the string (at $x=0$ ) nearest the gravitating object, with overdots denoting ordinary derivatives with respect to $t_{0}$, with $r \equiv \sqrt{y^{2}+z^{2}}$ being the approximate distance from the nearest point on the string to the gravitating object, and with $\gamma=\gamma\left(t_{0}\right)$ being a relativistic gamma-factor given by Eq. (27):

$$
\begin{aligned}
\dot{t} & \approx 1-\frac{2 \gamma M}{r} \dot{r} \dot{t}, \\
\dot{y} & \approx v_{0}-\frac{\pi \gamma\left(M^{2}-Q^{2}\right)}{8 r^{3}} \dot{t}^{2} y+\frac{2 \gamma M}{r} \dot{z}(z \dot{y}-y \dot{z}), \\
\dot{z} & \approx-\frac{\pi \gamma\left(M^{2}-Q^{2}\right)}{8 r^{3}} \dot{t}^{2} z-\frac{2 \gamma M}{r} \dot{z}(z \dot{y}-y \dot{z}) .
\end{aligned}
$$

In order for the string to remain nearly straight for all time, it is necessary that $r \gg \gamma M$, so Eq. (47) implies that under this condition, $\dot{t} \approx 1$, which can be inserted into Eqs. (48) and (49). Under this condition, we can also, to an excellent approximation, replace $\gamma\left(t_{0}\right)$ by the constant relativistic gamma-factor $\gamma_{0}=1 / \sqrt{1-v_{0}^{2}}$

The appearance of Eqs. (48) and (49) can be further simplified by defining the length scale

$$
L \equiv \sqrt{\frac{\pi\left(M^{2}-Q^{2}\right) \gamma_{0}}{8 v_{0}}}
$$

and the dimensionless parameter

$$
\alpha \equiv \frac{2 \gamma_{0} M}{L} \equiv \sqrt{\frac{32 M^{2} \gamma_{0} v_{0}^{3}}{\pi\left(M^{2}-Q^{2}\right)}}
$$


and by replacing the independent variable $t_{0}$ with $y_{0}=v_{0} t_{0}$. Having dispensed with the radial derivatives $\phi^{\prime}$ and $\psi^{\prime}$, we can now redefine the prime to mean differentiation with respect to $y_{0}$. Then Eqs. (48) and (49) take on the simplified forms

$$
\begin{aligned}
& y^{\prime} \approx 1-\frac{L^{2} y}{r^{3}}+\frac{\alpha L}{r^{2}} z^{\prime}\left(z y^{\prime}-y z^{\prime}\right), \\
& z^{\prime} \approx-\frac{L^{2} z}{r^{3}}+\frac{\alpha L}{r^{2}} y^{\prime}\left(z y^{\prime}-y z^{\prime}\right),
\end{aligned}
$$

with the initial conditions being that at $y_{0}=-\infty$, we have $y=-\infty$ and $z=b$.

Alternatively, one can convert these equations into polar coordinates such that $y=r \cos \theta$ and $z=r \sin \theta$ :

$$
\begin{aligned}
& r^{\prime} \approx \cos \theta-\frac{L^{2}}{r^{2}}-\alpha \operatorname{Lr} \theta^{\prime 2}, \\
& \theta^{\prime} \approx-\frac{\sin \theta}{r}+\frac{\alpha L}{r} r^{\prime} \theta^{\prime} .
\end{aligned}
$$

Another convenient pair of variables is $r$ and $c \equiv \cos \theta$, in which the equations take the simple form

$$
\begin{aligned}
r^{\prime} & \approx c-\frac{L^{2}}{r^{2}}+\frac{\alpha L r c^{\prime 2}}{1-c^{2}} \\
c^{\prime} & \approx-\frac{1-c^{2}}{r}+\frac{\alpha L}{r} r^{\prime} c^{\prime}
\end{aligned}
$$

Yet another pair, which is particularly useful for finding the solution when $\alpha \ll 1$, is $z$ and $c$. For these the equations of evolution are, using $s \equiv \sin \theta=\sqrt{1-c^{2}}$,

$$
\begin{aligned}
& z^{\prime} \approx-\frac{L^{2} s^{3}}{z^{2}}\left[1+\frac{\alpha z^{2}}{L s^{7}} c^{\prime}\left(s^{2} c z^{\prime}+z c^{\prime}\right)\right], \\
& c^{\prime} \approx-\frac{s^{3}}{z}\left[1+\frac{\alpha L}{s^{6}} c^{\prime}\left(s^{2} z^{\prime}+z c c^{\prime}\right)\right] .
\end{aligned}
$$

One can see from these equations that the only fixed point is at $(y, z)=(L, 0)$ or $(r, \theta)=(L, 0)$ or $(r, c)=(L, 1)$ or $(z, c)=(0,1)$. This represents a string that is marginally captured by the gravitating object. Its nearest point to the gravitating object, at $(x, y, z)=(0, L, 0)$, stays fixed in an unstable balance between the attractive force of the gravitating object in the negative $y$-direction toward the gravitating object location at the origin $(0,0,0)$ and the pull of the distant part of the string (at large $|x|$ ) toward the positive $y$-direction in which the distant part of the string continues moving at speed $v_{0}$.

We can provide a partial check on the approximations used so far by using the precise bending angle (5) for a static string in the Reissner-Nordstrom metric to calculate the relation between the point of nearest approach of a marginally captured 
string and the asymptotic velocity $v_{0}$. Looking at the string from a scale very large compared with $L$ (where the metric is essentially flat), the string will have a bend of $2 \delta$ near the gravitating object, given by Eq. (5) as a function of the coordinate $R_{0}$ of closest approach, and two kinks of $\delta$ each moving off at the speed of light into the part of the string that is parallel to the $x$-axis and moving in the $y$-direction with speed $v_{0}$.

By simple geometry, one can see that if the kinks are moving away from the gravitating object at the speed of light (one in our units) at an angle $\delta$ from the $x$-axis, then the component of their velocity in the $y$-direction is $\sin \delta$, which must be the same as $v_{0}$, the velocity of the string in the $y$-direction beyond the outwardmoving kink. (One can also easily see that in the laboratory frame in which the gravitating object is at rest, the component of the kink velocity in the $x$-direction is $\cos \delta$, but when one multiplies this by $\gamma_{0}=1 / \sqrt{1-v_{0}^{2}}=\sec \delta$, one gets that the kink also moves at the speed of light along the distant part that is parallel to the $x$-axis in the frame of that distant part of the string.)

Therefore, for a string marginally captured by a Reissner-Nordstrom black hole, the relation between the velocity $v_{0}$ of the distant straight part of the string and the stationary point of nearest approach to the black hole, at Schwarzschildean radial coordinate $r_{0}=R_{0}+M$, or at the conformally-flat radial coordinate $\rho_{0}$, is

$$
\begin{aligned}
v_{0} & =\sin \delta=\cos \left[k^{\prime} K(k)\right] \\
& =\cos \left[\frac{\sqrt{R_{0}^{2}-M^{2}+Q^{2}}}{R_{0}} K\left(\frac{\sqrt{M^{2}-Q^{2}}}{R_{0}}\right)\right] \\
& \approx \frac{\pi}{8} k^{2}+\frac{7 \pi}{128} k^{4} \\
& =\frac{\pi\left(M^{2}-Q^{2}\right)}{8 R_{0}^{2}}+\frac{7 \pi\left(M^{2}-Q^{2}\right)^{2}}{128 R_{0}^{4}} \\
& \approx \frac{\pi\left(M^{2}-Q^{2}\right)}{8 \rho_{0}^{2}}-\frac{\pi\left(M^{2}-Q^{2}\right)^{2}}{128 \rho_{0}^{4}} .
\end{aligned}
$$

This gives, for small $v_{0}$ or small $\left(M^{2}-Q^{2}\right) / \rho_{0}^{2}$,

$$
\rho_{0}=\left[1-\frac{v_{0}}{4 \pi}+O\left(v_{0}^{2}\right)\right] \sqrt{\frac{\pi\left(M^{2}-Q^{2}\right)}{8 v_{0}}},
$$

which indeed is $\rho_{0}=L$ to lowest order in $v_{0}$. For a Kerr-Newman black hole, the lowest-order term in $v_{0}$ (which goes as its inverse square root) is independent of the hole angular momentum and so is as given in Eq. (61), but the next-order term (which goes as the square root of $v_{0}$ ) will depend upon the square of the angular momentum, and upon the hole orientation relative to that of the string, as well as on $M^{2}-Q^{2}$, and so it is not accurately given by Eq. (61).

(The fact that the correction term goes linearly in $v_{0}$, rather than quadratically as $\gamma_{0}$ does, shows that for strings that become marginally trapped by the gravitating 
object at large $\rho_{0} \gg M$, the initial velocity $v_{0}$ must be so low, and $\gamma_{0}$ is so close to one, that for such strings it is meaningless to include the $\gamma_{0}$ factor in the definition (50) for the length scale $L$. However, it is included there to show how the effect of the $M^{2}-Q^{2}$ term in the metric depends on $v_{0}$ at all $v_{0}$, including values of $v_{0}$ very near unity or $\gamma_{0} \gg 1$, but still $\gamma_{0} \ll b / M$ so that the string stays approximately straight, as it scatters off the gravitating object without being captured.)

\section{Solution of the motion for very low velocity}

Now in principle, one can solve Eqs. (52)-(53) algebraically for $y^{\prime}(y, z)$ and $z^{\prime}(y, z)$, (54)-(55) for $r^{\prime}(r, \theta)$ and $\theta^{\prime}(r, \theta)$, (56)-(57) for $r^{\prime}(r, c)$ and $c^{\prime}(r, c)$, or (58)-(59) for $z^{\prime}(z, c)$ and $c^{\prime}(z, c)$, and then get the autonomous equation

$$
\begin{aligned}
& \frac{d z}{d y} \approx \frac{z^{\prime}(y, z)}{y^{\prime}(y, z)}, \\
& \frac{d r}{d \theta} \approx \frac{r^{\prime}(r, \theta)}{\theta^{\prime}(r, \theta)}, \\
& \frac{d r}{d c} \approx \frac{r^{\prime}(r, c)}{c^{\prime}(r, c)},
\end{aligned}
$$

or

$$
\frac{d z}{d c} \approx \frac{z^{\prime}(z, c)}{c^{\prime}(z, c)}
$$

The integral curve that matches the boundary condition $z=b$ at $y=-\infty$ or $c=-1$ then gives the trajectory in the $y-z$ plane of the point on the string $(x=0)$ closest to the gravitating object.

However, solving algebraically for the derivatives with respect to $y_{0}=v_{0} t_{0}$ always involves cubic equations whose explicit solutions are rather messy, and the resulting autonomous equations will almost certainly not be exactly separable in terms of elementary functions, so a different technique will employed, expanding in the dimensionless parameter $\alpha$ defined in Eq. (51).

In particular, we start with the zeroth-order solution in $\alpha$ by noting that when we drop the terms proportional to $\alpha$ in Eqs. (58)-(59), and take their ratio, we get

$$
\frac{d z}{d c} \simeq-\frac{L^{2}}{z}
$$

(Here and henceforth, I shall use $\simeq$ for the approximation that ignores correction terms of order $\alpha$, whereas I use $\approx$ for the better approximation that one is far from the gravitating object in units of its size, and that the string is at each moment of time nearly straight.) 
With the boundary condition that $z=b$ at $c=-1$, this simple differential equation has the solution

$$
z^{2} \simeq b^{2}-2 L^{2}(1+c) \equiv b^{2}-2 L^{2}(1+\cos \theta) \equiv b^{2}-4 L^{2} \sigma^{2}
$$

where

$$
\sigma \equiv \sin \left(\frac{\pi-\theta}{2}\right)=\cos \left(\frac{\theta}{2}\right)=\sqrt{\frac{1+c}{2}} \equiv \sqrt{\frac{1+\cos \theta}{2}} .
$$

We see from this that if $b>b_{\text {crit }} \simeq 2 L$, the trajectory goes from $c=-1(\theta=\pi$ or $\sigma=0)$ all the way to $c=+1(\theta=0$ or $\sigma=1)$ with $z^{2}$ remaining positive, so such a string is not captured but rather scatters from the gravitating object, with $z^{2}$ decreasing by an amount that is roughly $4 L^{2}$ (correct to zeroth order in $\alpha$ ), from $b^{2}$ to roughly $b^{2}-b_{\text {crit }}^{2} \simeq b^{2}-4 L^{2}$. However, for $b<b_{\text {crit }} \simeq 2 L, z^{2}$ approaches 0 at $c \equiv \cos \theta \simeq 2 b^{2} / b_{\text {crit }}^{2}-1, \sigma \simeq b / b_{\text {crit }}$, or

$$
\theta \simeq 2 \arccos \frac{b}{b_{\text {crit }}}
$$

This means that the string is captured by the gravitating object when it has an impact parameter $b<b_{\text {crit }} \simeq 2 L$.

During the final capture process by a black hole, when the string gets down to $r$ of the order of $M$, the string will no longer be nearly straight, so the trajectory of the nearest point on the string to the black hole will no longer be given by all of the equations above. However, then this point will just fall in nearly radially, so its path in the $y-z$ plane, though not the $y_{0}$-dependence of this path, will still be given approximately correctly by Eq. (67). For capture by other gravitating objects, the trajectory will differ when the string gets sufficiently near or within the object that the spacetime metric is no longer well approximated by the weak-field portion of the Reissner-Nordstrom metric Eq. (11).

In the intermediate case in which $b=b_{\text {crit }} \simeq 2 L$, the point on the string nearest the gravitating object will, asymptotically with large $t_{0}$ and with large $t$, approach the unstable fixed point which is approximately at $(y, z)=(L, 0)$, or, more precisely, at $z=0$ and at $y=\rho_{0}$ with $\rho_{0}$ given by the solution of Eq. (60). In this special case, one can integrate $d y_{0} / d c=1 / c^{\prime}$ explicitly in terms of elementary functions of $c$ (as opposed to elliptic integrals needed for a generic impact parameter). The results are slightly simpler in terms of $\sigma$ defined in Eq. (68) (the sine of half the angle from the negative $y$-axis, which thus goes from 0 when $y=-\infty$ to 1 when the string asymptotically approaches the fixed point on the positive $y$-axis), and there is an even simpler expression in terms of a new coordinate

$$
\psi \equiv \operatorname{gd}^{-1}\left(\frac{\pi-\theta}{2}\right)=\frac{1}{2} \ln \frac{1+\cos (\theta / 2)}{1-\cos (\theta / 2)},
$$


the inverse gudermannian of half the angle $\pi-\theta$ from the negative $y$-axis (not to be confused with the previous use of $\psi$ to denote an exponent in a metric component), which goes from 0 to $\infty$ and is defined so that

$$
\sigma \equiv \sin \left(\frac{\pi-\theta}{2}\right)=\tanh \psi .
$$

Then for the string which approaches with the critical impact parameter $b \simeq 2 L$, one gets

$$
\begin{aligned}
y_{0} & \simeq \frac{L}{2} \ln \left(\frac{1+\sigma}{1-\sigma}\right)-\frac{L}{\sigma}=L(\psi-\operatorname{coth} \psi), \\
t & \simeq t_{0}=\frac{y_{0}}{v_{0}} \simeq \frac{L}{2 v_{0}} \ln \left(\frac{1+\sigma}{1-\sigma}\right)-\frac{L}{v_{0} \sigma}=\frac{L}{v_{0}}(\psi-\operatorname{coth} \psi), \\
r & \simeq \frac{L}{\sigma}=L \operatorname{coth} \psi \\
y & \simeq L\left(\frac{-1+2 \sigma^{2}}{\sigma}\right)=L(2 \tanh \psi-\operatorname{coth} \psi) \simeq \frac{2 L^{2}}{r}-r \\
z & \simeq 2 L \sqrt{1-\sigma^{2}}=2 L \operatorname{sech} \psi \simeq \frac{2 L \sqrt{r^{2}-L^{2}}}{r} .
\end{aligned}
$$

\section{Discontinuities in the critical impact parameter}

For $v_{0} \nless 1$, where my analytic solution above of the critical capture is not valid, one would still have that the critical capture has the point on the string asymptotically approach the unstable fixed point at $z=0$ and at $y=\rho_{0}$ with $\rho_{0}$ given by the solution of Eq. (60). However, the relation between $b_{\text {crit }}$ and $\rho_{0}$ would not be so simple as the one above, $b_{\text {crit }} \simeq 2 \rho_{0}$ for $v_{0} \ll 1$. For example, for a Schwarzschild black hole $(Q=0)$ and for $v_{0}=1$, Eq. (60) gives $R_{0}=M$, and then Eq. (11) shows that $\rho_{0}=M / 2$. On the other hand, $b_{\text {crit }}=3 \sqrt{3} M$ at this limit of the speed of light

[2], so there $b_{\text {crit }}=6 \sqrt{3} \rho_{0}$, with the ratio being $3 \sqrt{3}$ times larger at the speed of light than at very low velocities.

To get a ratio that does not vary quite so much, one might instead use the Schwarzschildean or circumferential radial coordinate of the unstable fixed point, which by Eq. (11) is

$$
r_{0}=\rho_{0}\left(1+\frac{M}{2 \rho_{0}}\right)^{2}-\frac{Q^{2}}{4 \rho_{0}} .
$$

For large $\rho_{0} / M\left(v_{0} \ll 1\right), r_{0} \sim \rho_{0}$, but for $\rho_{0}=M / 2\left(v_{0}=1\right), r_{0}=2 M=4 \rho_{0}$ for a Schwarzschild black hole. Then one could define the ratio

$$
F\left(v_{0}\right) \equiv \frac{b_{\text {crit }}}{2 r_{0}}
$$


which goes from 1 at $v_{0}=0$ to $(3 / 4) \sqrt{3} \approx 1.299038$ at $v_{0}=1$ for a test string in the Schwarzschild geometry.

An interesting point about $F\left(v_{0}\right)$, which is a dimensionless number of order unity (a function of the string initial velocity $v_{0}$, and of $(Q / M)^{2}$ for a Reissner-Nordstrom black hole) that parametrizes the critical impact parameter for the capture of a infinite test string by a black hole, is that I would expect it (and hence also the critical impact parameter $b_{\text {crit }}$, since $r_{0}$ is a continuous function of $v_{0}$ ) to have an infinite number of discontinuities as $v_{0}$ is increased from 0 to 1 . These discontinuities should arise from the discreteness of the number of times the closest point on the string (at $x=0$ ) wraps around the black hole before this point approaches the unstable fixed point characterizing the critical capture solution at a particular $v_{0}$. At very low $v_{0}$ we found above that the angle $\theta$ of this closest point on the string from the positive $y$-axis decreased monotonically from $\pi$ down to 0 in approaching the unstable fixed point, but for a sufficiently larger values of $v_{0}$, I would expect it to decrease to $-2 \pi n$ for some positive integer $n$ that depends discontinuously on $v_{0}$ and characterizes how many times the string wraps around the black hole before it makes its final asymptotic approach to the unstable fixed point. As $v_{0}$ approaches $1, n$ should approach $\infty$, as the string wraps around the black hole an infinite number of times before approaching the unstable fixed point characterizing the final marginally bound string.

Unless there are some surprising cancellations, I would expect that $F\left(v_{0}\right)$, and hence $b_{\text {crit }}\left(v_{0}\right)=F\left(v_{0}\right) r_{0}\left(v_{0}\right)$, to have a discontinuity each time $v_{0}$ crossed a discontinuity of the wrapping integer $n$, with an infinite number of such discontinuities occurring as one approaches the speed of light $\left(v_{0}=1\right)$. Indeed, when I voiced this prediction, De Villiers [12] confirmed that he has seen a suggestion of it in his numerical calculations [13].

The situation would also be complicated by the fact that cusps and loops form in the string when $v_{0}$ is large enough [1]. To get a simple unique definition for $b_{\text {crit }}\left(v_{0}\right)$, one could assume that any loops that form do not break off, in which case it seems clear that the marginal capture behavior is indeed when the point on the string approaches the unstable fixed point. However, if one allowed loops to break off the string, one would have to specify when the loops break off and whether capture was defined to be the case in which at least one such loop ended up orbiting or falling into the black hole, or whether the piece connected to infinity itself has to become bound to the hole. In the former specification I am not certain that one would necessarily get a critical impact parameter that is discontinuous in the velocity (as I am arguing that it is when one does not allow loops to break off, and which it also should be if capture is defined so that the piece of the string connected to infinity itself has to become bound to the hole). However, it is not obvious to me how to come up with a definition that would make the critical impact parameter a continuous function of the string initial velocity $v_{0}$. In any case in which loops of string break off, they 
would carry away momentum and alter the critical impact parameter in a way that would depend upon when the loops break off.

\section{$7 \quad$ First-order corrections at finite velocity}

Now return to the analysis of low initial string velocities, $v_{0} \ll 1$, where the wrapping integer $n$ is zero and the critical impact parameter $b_{\text {crit }}\left(v_{0}\right)$ is a continuous function of $v_{0}$. Let us evaluate the first-order corrections in the dimensionless parameter $\alpha$ defined by Eq. (51). Define the quantity

$$
B \equiv \frac{z^{2}}{2 L^{2}}+c=\frac{z^{2}}{2 L^{2}}+2 \sigma^{2}-1 \simeq \frac{b^{2}}{2 L^{2}}-1,
$$

which to zeroth order in $\alpha$ is thus constant. From Eqs. (52)-(59), one can deduce that the derivative of $B$ with respect to $c \equiv \cos \theta \equiv 2 \sigma^{2}-1$ is

$$
\frac{d B}{d c} \approx-\alpha\left(\frac{r}{L} y^{\prime}-\frac{L}{r} r^{\prime}\right)
$$

Evaluate $r^{\prime}$ and $y^{\prime}$ to zeroth order in $\alpha$ to get

$$
\frac{d B}{d c} \cong-\alpha\left(\frac{r}{L}-\frac{2 L c}{r}+\frac{L^{3}}{r^{3}}\right),
$$

where the $\cong \operatorname{sign}$ means that the equation is accurate through first order in $\alpha$ (and thus better than the $\simeq$ approximations, which are only accurate to zeroth order in $\alpha$, but not so good as the $\approx$ approximations, which are accurate for all $\alpha$ and which essentially only require that the string configurations be nearly straight).

Next, insert the zeroth-order solution for $r(c)$ into the right hand side of Eq. (81). To simplify the result, replace the independent variable $c$ with $\sigma$ and define the dimensionless parameter

$$
k \equiv \frac{b_{\text {crit }}}{b} \simeq \frac{2 L}{b}
$$

not to be confused with the previous use of $k$ as defined in Eq. (6); I use the same letter because this $k$, like the previous one, will be used as the modulus of a complete elliptic integral. Then one gets

$$
\frac{d B}{d \sigma} \cong-\frac{4 \alpha\left(1-6 k^{2} \sigma^{4}+4 k^{2} \sigma^{6}+k^{4} \sigma^{8}\right)}{k\left(1-k^{2} \sigma^{2}\right)^{3 / 2} \sqrt{1-\sigma^{2}}} .
$$

Consider the case in which $k<1$, so that the impact parameter $b$ is greater than the critical impact parameter $b_{\text {crit }} \simeq 2 L$ and so that the string scatters without being captured, with $z$ remaining positive as $\sigma=\cos \theta / 2$ goes from 0 at $\theta=\pi$ and $y=-\infty$ to 1 at $\theta=0$ and $y=+\infty$. Then integrating Eq. (83) from $\sigma=0$ to $\sigma=1$ 
gives the change in $B$ in terms of the complete elliptic integrals $K(k)$ (of the first kind) and $E(k)$ (of the second kind) with modulus $k$ :

$$
\Delta B \cong \frac{32 \alpha}{15 k^{5}}\left[\left(2-3 k^{2}+k^{4}\right) K(k)+\left(-2+2 k^{2}-2 k^{4}\right) E(k)\right] .
$$

One can then use this result to get the final value of $z^{2}$ as $y$ goes to $+\infty$ :

$$
z_{f}^{2}=b^{2}-4 L^{2}+2 L^{2} \Delta B
$$

When the impact parameter is very near the critical impact parameter, the complementary modulus

$$
k^{\prime} \equiv \sqrt{1-k^{2}} \equiv \sqrt{1-b_{\text {crit }}^{2} / b^{2}}
$$

is very small, and from the form of $K(k)$ and $E(k)$ for small $k^{\prime}$, one gets from Eq. (84)

$$
\Delta B \cong-\frac{64 \alpha}{15}\left[1+\frac{5}{4} k^{\prime 2}-O\left(k^{\prime 4} \ln \frac{\text { const. }}{k^{\prime}}\right)\right] .
$$

Taking the limit of this when the impact parameter reaches the critical impact parameter, so that $k^{\prime}=0$, and inserting the result back into Eq. (85) with $z_{f}=0$ gives the critical impact parameter to first order in $\alpha$ :

$$
b_{\text {crit }} \cong 2 L+\frac{32}{15} \alpha L \approx \sqrt{\frac{\pi\left(M^{2}-Q^{2}\right)}{2 v_{0}}}+\frac{64}{15} M v_{0} .
$$

Here I have dropped the factors of $\gamma_{0}$, since these expressions are only valid for $b_{\text {crit }} \gg M$, and this implies that $v_{0} \ll 1$ so that $\gamma_{0} \approx 1$.

It is amusing that if one evaluates the last expression on the right hand side of Eq. (88) for a Schwarzschild black hole $(Q=0)$ at $v_{0}=1$ (which is far outside its range of validity, which is for $\left.v_{0} \ll 1\right)$, one gets $(\sqrt{\pi / 2}+64 / 15) M \approx 5.519981 M$, which is not too far from the actual [2] $b_{\text {crit }}=3 \sqrt{3} M \approx 5.196152 M$ at the speed of light. Since Eq. (88) is only valid for $b_{\text {crit }} \gg M$, it probably misses a $v_{0}$-independent term of order $M$. It is therefore tempting to add such a term to give the right answer at $v_{0}=1$ and conjecture that a crude approximation for the critical impact parameter for a straight string moving at any initial velocity $v_{0}$ in the field of a Schwarzschild black hole is

$$
\begin{aligned}
b_{\text {crit }} & \stackrel{?}{\sim}\left[\sqrt{\frac{\pi}{2 v_{0}}}-\left(\sqrt{\frac{\pi}{2}}+\frac{64}{15}-\sqrt{27}\right)+\frac{64}{15} v_{0}\right] M \\
& \approx\left(1.253314 v_{0}^{-1 / 2}-0.323828+4.266667 v_{0}\right) M .
\end{aligned}
$$

Here the $\stackrel{?}{\sim}$ emphasizes that this is only a very crude guess that gives a good approximation at both $v_{0} \ll 1$ and at $v_{0}=1$, but it has by no means been derived 
at intermediate velocities, and in this paragraph the $\approx$ just means the numerical approximation of using a finite decimal approximation for the numbers given exactly before the $\approx$. Of course, this crude guess is also continuous and so misses the infinite number of discontinuities that were predicted above for $b_{\text {crit }}\left(v_{0}\right)$.

It is also amusing to note that this crude guess (89) gives a minimum critical impact parameter of

$$
\begin{aligned}
b_{\text {crit min }} & \stackrel{?}{\sim}\left[6\left(\frac{\pi}{15}\right)^{1 / 3}-\sqrt{\frac{\pi}{2}}-\frac{64}{15}+\sqrt{27}\right] M \\
& \approx 3.239349 M
\end{aligned}
$$

at $v_{0} \stackrel{?}{\sim}(225 \pi)^{1 / 3} / 32 \approx 0.278373$. Numerical calculations [2] do show that the critical impact parameter decreases as $v_{0}$ is reduced below unity, and they hint of the rise at lower impact parameters that must occur for Eq. (88) to be valid there, but it remains to be seen how accurate the crude guess of Eq. (90) is for the minimum critical impact parameter and the velocity $v_{0}$ at which it occurs.

I should warn that although Eq. (88) attempts to give the term in the critical impact parameter that is linear in $v_{0}$, I would suspect that this term, even with a $v_{0}$-independent term that I suspect may be present but which I do not know how to evaluate, does not give the leading correction to the dominant term that goes as $v_{0}^{-1 / 2}$. We have seen from Eq. (61) that the location of the unstable critical point for a string that is just marginally captured by a black hole has a correction term to $L$ that goes as $v_{0}^{1 / 2}$, and I would suspect that $b_{\text {crit }}$ also has a correction term going as $v_{0}^{1 / 2}$, though I do not know what it is. Therefore, I suspect that for low velocities $v_{0}$, the critical impact parameter for a Schwarzschild black hole may be expanded as

$$
b_{\text {crit }}=\left[\sqrt{\pi / 2} v_{0}^{-1 / 2}+O(1)+O\left(v_{0}^{1 / 2}\right)+O\left(v_{0}\right)+O\left(v_{0}^{3 / 2}\right)\right] M .
$$

The only thing I can assert with confidence is that I have derived the coefficient of the leading term, but I do not know the coefficients of the $O(1)$ and $O\left(v_{0}^{1 / 2}\right)$ terms that I suspect are present. Eq. (88) might give the correct $O\left(v_{0}\right)$ term, but I am also not certain that there are no other $O\left(v_{0}\right)$ terms that have been missed in the approximations that I have used. In other words, the only firm conclusion that I can make from the approximations that I have made is that in the limit of low velocity $v_{0}$ for a straight test string impinging upon a Kerr-Newman black hole, or for any other gravitating object that is very small in comparison with the string impact parameter,

$$
\lim _{v_{0} \rightarrow 0} v_{0}^{1 / 2} b_{\text {crit }}=\sqrt{\frac{\pi\left(M^{2}-Q^{2}\right)}{2}} .
$$

Next, go back to Eq. (84) and evaluate it when the impact parameter $b$ is much greater than the critical impact parameter $b_{\text {crit }}$, so that $k \equiv b_{\text {crit }} / b \ll 1$. Then

$$
\Delta B \cong-\frac{2 \pi \alpha}{k}+\frac{\pi \alpha k}{2} \cong-\pi \alpha\left(\frac{b}{L}-\frac{L}{b}\right)
$$


However, I suspect that my approximations have missed out terms that are larger than the second term of the right hand side of Eq. (93), which is much smaller than the first term, so I shall henceforth drop the second term.

Inserting the resulting $\Delta B$ from Eq. (93) for $b \gg 2 L$ into Eq. (85), and taking the square root to the same level of approximation, gives the final height of the string, over the plane through the gravitating object location that is parallel to the string's original extension and motion, as

$$
z_{f} \cong b-\frac{2 L^{2}}{b}-\pi \alpha L \approx b-\frac{\pi\left(M^{2}-Q^{2}\right)}{4 v_{0} b}-2 \pi M \gamma_{0} v_{0}
$$

Here I have dropped the $\gamma_{0}$ factor from the second term, since it is only significant in comparison with the third term if $v_{0} \ll 1$, which gives $\gamma_{0} \approx 1+v_{0}^{2} / 2 \approx 1$. However, I have retained the $\gamma_{0}$ factor in the third term, since it is actually valid for all values of the celerity $\gamma_{0} v_{0}$. This formula is precisely the same as that derived independently in $[3]$.

One might think that Eq. (94) is only valid for $\alpha \ll 1$, which one can easily see from the definition of $\alpha$ in Eq. (51) implies that $v_{0} \ll 1$. Indeed, my derivation did assume this when I expanded in powers of $\alpha$ and kept only the lowest nontrivial power. However, the leading term in $\Delta B$ at large $b / L$, actually comes from just the first term of Eq. (80), which is then simply

$$
\frac{d B}{d c} \approx-\alpha\left(\frac{r}{L} y^{\prime}\right)
$$

without using any approximation that $\alpha \ll 1$. Continuing at large $b / L$, for the right hand side it is a sufficiently good approximation to set $z \approx b$ (so $r \approx b / \sin \theta=$ $b / \sqrt{1-c^{2}}$ and $y^{\prime} \approx 1$ ), in which case Eq. (95) becomes

$$
\frac{d B}{d c} \approx-\frac{\alpha b}{L \sqrt{1-c^{2}}}
$$

which, when integrated from $c=-1$ to $c=1$, gives

$$
\Delta B \approx-\frac{\pi \alpha b}{L}
$$

independent of the size of $\alpha$. This leads to Eq. (94) for all values of $v_{0}$, so long as $b \gg b_{\text {crit }} \simeq 2 L$.

Even more simply, one may derive Eq. (94) directly from Eq. (53) by putting $y \approx y_{0}$ and $z \approx b$ (and hence $y^{\prime} \approx 1$ and $z^{\prime} \approx 0$ ) on the right hand side and integrating.

By using these results for $b$ near $b_{\text {crit }}$ and for $b \gg b_{\text {crit }}$, we can replace Eq. (85), with its $\Delta B$ given by Eq. (84) in terms of complete elliptic integrals, with a simpler explicit expression that has almost as much relative accuracy for $z_{f}^{2}$ (except when it 
is very tiny) and for $z_{f}^{2}-b^{2}$, though some relative accuracy is lost for the difference between $z_{f}^{2}$ and its zeroth-order (in $\alpha$ ) approximation $b^{2}-4 L^{2}$ when this difference is small, which occurs when $b$ is not much larger than $2 L$. Namely, we may write

$$
z_{f}^{2} \approx b^{2}-\frac{\pi\left(M^{2}-Q^{2}\right)}{2 v_{0}}-4 \pi M \gamma_{0} v_{0} b
$$

which is an excellent approximation so long as $b \gg M \gamma_{0}$ and so long as the resulting $z_{f}^{2}$ it gives is positive and much larger than the magnitude of the third (last) term on the right hand side.

To see that this is very nearly what the more accurate Eq. (85) gives, we can consider various cases. First, consider the case in which the magnitude of the second term on the right hand side of Eq. (98) is larger than the magnitude of the third term. This occurs for $b<\left(M^{2}-Q^{2}\right) /\left(8 M \gamma_{0} v_{0}^{2}\right)$. Since we are requiring $M \ll b$, this case implies that $v_{0} \ll \sqrt{1-Q^{2} / M^{2}}$, a low velocity, justifying the omission of the $\gamma_{0}$ factor that is in the second term of the right hand side of Eq. (85) $\left(-4 L^{2}\right)$ but which is dropped in the simplified second term of Eq. (98).

Then the third term of the right hand side of Eq. (85) $\left(+2 L^{2} \Delta B\right)$, which is always of the same order of magnitude as the third term of the right hand side of Eq. (98) $\left(-4 \pi M \gamma_{0} v_{0} b\right)$ but which differs by a factor of order unity that is significantly different from unity when $b$ is not very large compared with $b_{\text {crit }} \sim 2 L$, will be much less than the second term unless $b$ nearly saturates the inequality given above. If $b$ does nearly saturate this inequality, one can easily see that one must have $b \gg b_{\text {crit }}$. Therefore, when the modulus $k$ of the elliptic functions in Eq. (84) for $\Delta B$ is of order unity $\left(b \gg b_{\text {crit }}\right)$, the third term of the right hand side of Eq. (85) $\left(+2 L^{2} \Delta B\right.$ ), and the third term of the right hand side of Eq. (98) $\left(-4 \pi M \gamma_{0} v_{0} b\right)$, are both very small compared with the second term, and so they may both be ignored, even though they may differ by a factor of order unity (e.g., by a factor of about $15 \pi / 16$ at $b=b_{\text {crit }}$ ). On the other hand, when the third terms of the right hand sides of Eqs. (85) and (98) are not negligible in comparison with the second term, one has $b \gg b_{\text {crit }}$ and hence $k \ll 1$, so that these two third terms are very nearly the same.

In the opposite case, in which the magnitude of the second term on the right hand side of Eq. (98) is smaller than the magnitude of the third term, which occurs for $b>\left(M^{2}-Q^{2}\right) /\left(8 M \gamma_{0} v_{0}^{2}\right)$, by invoking our assumption $b \gg M \gamma_{0}$ one can easily see that in this case $k^{2} \approx 4 L^{2} / b^{2}=\pi\left(M^{2}-Q^{2}\right) \gamma_{0} /\left(2 v_{0} b^{2}\right) \ll 1$, so $\Delta B$ is well approximated by $-\pi \alpha b / L$ and the third terms of the right hand sides of Eqs. (85) and (98) are very nearly the same.

In other words, the third term of the right hand side of Eq. (98) is not always an accurate approximation to the third term of the right hand side of Eq. (85), but it is when either of these two terms makes any significant difference to $z_{f}^{2}-b^{2}$.

Eq. (98) thus gives a good approximation for the final height $z_{f}$ (above the $z=0$ plane containing the center of mass of the gravitating object and parallel to the string's initial straight extent in the $x$-direction and motion with speed $v_{0}$ in 
the $y$-direction) in terms of the initial height $z_{i}=b$, the speed $v_{0}$, and the mass $M$ and charge $Q$ of the gravitating object, so long as the string stays in the electrovac weak-field region outside the object, far enough away that one can ignore the metric deviations from sphericity due to the angular momentum and multipole moments of the gravitating object, and so long as $b \gg M \gamma_{0}$ so that the third term of Eq. (98) is small compared with its first term $\left(b^{2}\right)$.

\section{Formulas for arbitrary velocity}

It would be nice to remove the restriction to $\gamma_{0} \ll b / M$ (which is implied by the method used above in order that the string remain nearly straight during its entire scattering), and indeed one can do so by trivially extending [8] to arbitrarily large celerity the results of [3], that to linear order in $M$ (i.e., ignoring the $M^{2}-Q^{2}$ terms that are only negligible when $\left.v_{0}^{2} \gg(M / b)\left(1-Q^{2} / M^{2}\right)\right)$, the final deflection is simply $z_{f}-b \sim-2 \pi M \gamma_{0} v_{0}$ (assuming that the string stays in the far-field region where the gravitational field is not only weak, $b \gg M$, but also nearly spherically symmetric, and assuming that any loops which may form in the string do not break off to carry away momentum).

The argument that this formula is valid for arbitrarily large celerities (and hence arbitrarily large $\gamma_{0}$, even for $\left.\gamma_{0}>b / M\right)$ is, in brief, the following [4, 8]: First, if one has an infinitely long string of tension $\mu$ passing far from a gravitating object $\left(b \gg M / v_{0}^{2}\right.$, and also $b v_{0}^{2}$ much larger than the scale given by any distortions from the spherical electrovac Reissner-Nordstrom metric), in the test string approximation $\mu \ll M \gamma_{0} v_{0} / b$ [4] so that the gravitating object does not accelerate significantly from the back reaction of the string, then to first order in $M$ (which dominates when the inequalities above hold), the momentum transferred from a gravitating object to the string is $4 \pi \mu M \gamma_{0} v_{0}$, perpendicular to the motion of the string and toward the side on which the object passes the string, for arbitrarily large $\gamma_{0}$. Second, it is easy to see [4, 8] that an infinitely long, initially straight, test string in flat spacetime undergoes a total transverse displacement from its otherwise uniform motion that is simply the transverse momentum transferred to the string divided by $2 \mu$, whether or not cusps or loops form (so long as they do not break off to carry away momentum from the infinitely long connected portion of the string).

One can easily see that this formula for the final deflection is indeed what Eq. (98) gives when one ignores the second term (with the $M^{2}-Q^{2}$ factor) and takes $b \gg M \gamma_{0}$ so that the third term is small in comparison with the first term. But when one does not have $b \gg M \gamma_{0}$, Eq. (98) is not accurate. E.g., if the third term is larger than the first term, it would nonsensically give a negative $z_{f}^{2}$, even though the string scatters and is not captured as it would be if the second term were larger than the first term (when a negative expression for $z_{f}^{2}$ does have the meaning that something went wrong with the assumption that the string scattered). 
However, one can easily combine Eq. (98), which is valid for $b \gg M \gamma_{0}$, with $z_{f}-b \sim-2 \pi M \gamma_{0} v_{0}$ [3], which is valid for $b \gg M / v_{0}^{2}$ for arbitrarily large $v_{0}$ [ [B], as briefly argued above, to get

$$
z_{f} \approx b \sqrt{1-\frac{\pi\left(M^{2}-Q^{2}\right)}{2 b^{2} v_{0}}}-2 \pi M \gamma_{0} v_{0},
$$

which is valid for all test string velocities $v_{0}$ so long as $b \gg M$ so that the string stays in the weak-field regime (and also assuming that the gravitational field is electrovac and nearly spherically symmetric in this regime).

Therefore, by devising an approximation that works for all infinitely long test strings that pass by a gravitating object at sufficiently large distance that that stay nearly straight at each moment of time that they are influenced significantly by the gravitational field of the object, we can extend the analysis of [3] to arbitrarily low and high velocities. We thereby find the critical impact parameter for capture at low velocity,

$$
b_{\text {crit }}=M\left(\sqrt{(\pi / 2)\left(1-Q^{2} / M^{2}\right) / v_{0}}+O(1)+O\left(v_{0}^{1 / 2}\right)+\frac{64}{15} v_{0}\right)
$$

(though I have not ruled out the possibility of other $O\left(v_{0}\right)$ terms that could change the coefficient 64/15), and we find that if the actual impact parameter, $z_{0}=b$, is greater than the critical impact parameter $b_{\text {crit }}$, the final height of the string, $z_{f}$, is given to a good approximation by Eq. (99) for all velocities $v_{0}$ when $b \gg M$.

\section{Acknowledgments}

I thank Jean-Pierre De Villiers and Valeri Frolov for pointing out to me that the gravitational capture of slowly moving test strings was an unsolved problem, and, shortly after my calculations were completed and written up, for giving me their paper [3] which independently derived the perturbative scattering of strings and which agrees with the limiting case of my results in which one takes the impact parameter to be much larger than the critical impact parameter for capture. I also thank them for further discussions, for guiding me to some of the literature on this subject, and for comments on my manuscript. This work was supported in part by the Natural Sciences and Engineering Research Council of Canada. 


\section{References}

[1] S. Lonsdale and I. Moss, Nucl. Phys. B298, 693 (1988).

[2] J.-P. De Villiers and V. Frolov, "Gravitational Capture of Cosmic Strings By a Black Hole," University of Alberta preprint Thy 14-97 (1997), gr-qc/9711045.

[3] J.-P. De Villiers and V. Frolov, "Scattering of Straight Cosmic Strings by Black Holes: Weak Field Approximation," University of Alberta preprint Thy 04-98 (1998), gr-qc/9804087.

[4] D. N. Page, "Nonlinear Gravitational Interactions with Slow Cosmic Strings," University of Alberta preprint Thy 06-98 (1998), in preparation.

[5] V. P. Frolov, V. D. Skarzhinsky, A. I. Zelnikov, and O. Heinrich, Phys. Lett. B224, 255 (1989); B. Carter and V. P. Frolov, Class. Quant. Grav. 6, 569 (1989).

[6] A. Vilenkin, Phys. Rev. Lett. 46, 1169 and 1496 (1981).

[7] See, for example, C. W. Misner, K. S. Thorne, and J. A. Wheeler, Gravitation (W. H. Freeman, San Francisco, 1973).

[8] D. N. Page, "Gravitational Scattering of Distant Strings with Very High Celerity," University of Alberta preprint Thy 07-98 (1998), in preparation.

[9] Y. Nambu, in Proceedings of the International Conference on Symmetries \&6 Quark Models, Wayne State University (1969); Lectures at the Copenhagen Summer Symposium (1970).

[10] T. Goto, Prog. Theor. Phys. 46, 1560 (1971).

[11] B. Carter, Phys. Lett. B224, 61, (1989); J. Geom. Phys. 8, 53 (1992); Class. Quant. Grav. 9, 19 (1992); Phys. Rev. D10, 4835 (1993); in Formation and Interactions of Topological Defects, eds. R. Brandenberger and A.-C. Davis (Plenum, New York, 1995), p. 304, hep-th/9611054; "Brane Dynamics for Treatment of Cosmic Strings and Vortons," Tlaxcala lecture notes, 2nd Mexican School on Gravitation and Mathematical Physics, 1-7 Dec. 1996, eds A. Garcia, C. Lammerzahl, A. Macias, and D. Nunez, hep-th/9705172.

[12] J. P. De Villiers, private communication.

[13] J.-P. De Villiers and V. Frolov, "Gravitational Scattering of Cosmic Strings By a Black Hole," in preparation. 